\title{
THE POST-OPERATIVE MANAGEMENT, INCLUDING THE MANAGEMENT OF SOME OF THE COMMON COMPLICATIONS, OF PARTIAL GASTRECTOMY
}

\author{
By O. E. OwEN, M.B., F.R.C.S.
}

Senior Surgical Registrar, St. Fames' Hospital, London, S.W.12

The care of the patient after partial gastrectomy is a highly individualized problem which will be rendered less complicated by careful pre-operative assessment and preparation, a meticulously performed operation by an experienced surgeon and by expert anaesthesia.

\section{Pre-operative Management}

It is advisable to admit cases for gastrectomy three days prior to operation. This enables the patient to gain confidence in the staff, to become familiar with the surroundings and to be given a general explanation as to what to expect in the post-operative period. Instruction in deep breathing exercises can be given and the haemoglobin and blood group estimated. In the presence of any acute respiratory or naso-pharyngeal infection it is wiser to delay operation at least Io days. Patients with disturbed physiological balance as a result of pyloric stenosis, bleeding or'starvation, will need a longer period of preparation. A preliminary period of bed-rest is of great value when there is acute exacerbation of an ulcer; it allows oedema around the ulcer to subside, the fibrotic tissues hold stitches better and the surgery is easier.

On the eve of operation a stomach tube is passed. Occasionally unsuspected gastric retention is brought to light by this procedure. The operative field is prepared and an enema given. After a light evening meal a sedative assures a restful night's sleep.

On the day of operation the skin is prepared again. The stomach is emptied one and a half hours before operation, using a trans-nasal Ryle's or oesophageal tube. The anaesthetic premedication is given. The stomach is aspirated again immediately before leaving the ward and the tube left in place.

\section{Types of Operation Performed}

The operation performed may be simple: that is, as for ulcer; or radical (removing perigastriic. tissues), as for cancer.

These gastrectomies can be classified as follows

\begin{tabular}{|c|c|}
\hline Simple & RADICAL \\
\hline $\begin{array}{l}\text { Lower Partial ('Gastro- } \\
\text { duodenectomy') }\end{array}$ & $\begin{array}{c}\text { Lower Partial ('Gastro- } \\
\text { duodenectomy') }\end{array}$ \\
\hline $\begin{array}{l}\text { Gastro-duodenal anastomosis } \\
\text { (Billroth I) } \\
\text { Gastro-jejunal anastomosis } \\
\text { (1) Antecolic Valved Balfour } \\
\text { (proximal jejunum to } \\
\text { lesser curve) } \\
\text { (2) Antecolic Valved Moyni- } \\
\text { han (proximal jejunum } \\
\text { to greater curve) }\end{array}$ & $\begin{array}{l}\text { In view of the glang } \\
\text { clearance around cafe } \\
\text { dia, even lower pattial } \\
\text { gastrectomymeans } \\
\text { of most of vagal fibses }\end{array}$ \\
\hline $\begin{array}{l}\text { Upper Partial ('Oesophago- } \\
\text { gastrectomy') } \\
\text { Necessarily includes a vago- } \\
\text { tomy }\end{array}$ & 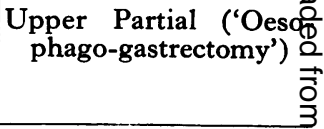 \\
\hline
\end{tabular}

The patient is received back from the theatre into a warmed bed. The pulse rate and bloot pressure are recorded hourly, the stomach emptie $\overline{\text { A }}$. by aspiration and the volume and nature of the contents noted. The aspirate usually contains. ' coffee grounds' at this stage. Gastric aspiration serves to draw off bloody fluid and swallowed air? which would lead to gastric distension and prolong post-operative paresis, particularly if vagotomizeds It will also reveal bleeding from the suture line The stomach is aspirated hourly for the first 2 y hours and the volume is recorded on a fluid balance chart. Unless the patient is already on an intrag venous infusion, a slow rectal drip of tap water is commenced and continued for 24 hours. If the patient sweats or the weather is hot, intravenoug fluids may be used. Any untoward sign must bథ promptly reported, interpreted, evaluated and acted upon. The onset of shock shortly after? operation may be due to excessively severe surgical 
procedure, bleeding, lung collapse or pain. When the patient is conscious he is placed in a sitting-up position and encouraged to breathe deeply and to cough. At this stage morphia is necessary to relieve pain and small sips of cold water can be given to allay thirst. To ensure a restful night morphia is given, but not more often than four-hourly.

\section{First Post-operative Day}

At 8 a.m. a glass of cold water $(8 \mathrm{oz}$.) is given to drink and five minutes later the stomach is emptied by aspiration. This serves as a stomach wash-out and at this stage the contents are usually ' coffee grounds' or a few clots and mucus, and sometimes bile. The tube is not kept in after this, but is reinserted when indicated by nausea, epigastric discomfort, bloating or repeated belching. Breathing exercises are carried out with the patient in the sitting-up position, lying flat, on one side and then on the other. If the bronchial secretions are excessive, the foot of the bed is elevated for a period of ro minutes with the patient on each side and . then he is encouraged to cough. This exercise is most effective if done after an analgesic has been given.

If there is doubt about full aeration of the lungs, an X-ray should be taken. A new shadow in the lung fields may indicate collapse, pneumonia or an infarct. In the case of lung collapse, an intravenous injection of nikethamide is given and the patient encouraged to cough. If the lung does not expand with this measure, tracheal and bronchial aspiration by trans-nasal catheter on strong intermittent suction is performed. Antibiotic therapy is started if pneumonia is suspected. After this course of breathing exercises the patient is made comfortable in a sitting-up position and a hot, sweetened cup of tea is brought. This is a just reward for his trials and a great booster for the morale. The stomach tube is passed three times altogether in the day and the breathing exercises carried out as well. Audible intestinal peristaltic activity usually returns within 36 to 48 hours after operation and on account of this a cautious resumption of oral feeding is indicated. Cold water or fruit juices are given in 4- to 6-oz. doses two-hourly after the first 24 hours. If at any time the patient has hiccoughs, a feeling of fullness or a cramp-like pain in the upper abdomen, the gastric tube is passed, the stomach emptied and the tube left in position. Rectal or intravenous saline may be recommended. A urinary output of 30 to $50 \mathrm{oz}$. in 24 hours usually indicates that the body is receiving sufficient fluids and the appearance of the tongue and elasticity of the skin give a guide to the state of hydration. Allowance must be made for extra loss of water through the skin in hot weather. To avoid overloading the circulation, particularly in cases with chronic bronchitis and heart disease, it is better to keep the patient ' a little on the dry side.'

\section{Subsequent Management}

If progress is satisfactory, aspiration can cease on the third day except if the patient is uncomfortable in the evenings, or if he cannot face breakfast in the mornings. Aspirations at this stage are more often required after the Billroth $\mathrm{I}$ operation. Fluids by mouth are increased so that the patient is able to take hourly milk feeds and liquids freely. Mild colicky lower abdominal pain, indicating the return of normal bowel activity, is usually relieved by a flatus tube and a small soap and water enema.

By the fourth day the diet is increased by adding milk, junkets, jellies, toast and eggs. The patient is allowed up for short periods and is walking to the toilet. Early ambulation by allowing the patient to sit out of bed on the first and second days, together with the deep breathing exercises, help to prevent venous stagnation in the limbs, with consequent risk of phlebothrombosis and pulmonary embolism. A daily inspection of the legs is carried out. A low-grade temperature with painful, tense or swollen calves indicate the need for active treatment with anticoagulants. Pulmonary embolism is heralded by chest pain and haemoptysis.

By the fifth day minced chicken, fish and nutrient soups are given and gradually the food is increased, so that by the tenth day a full normal diet is being enjoyed.

Skin clips or stitches are usually removed on the sixth or seventh day and the stay in hospital is 14 days for the average uncomplicated case.

\section{Management After Emergency Operations}

Post-operative care following emergency partial gastrectomy for bleeding or perforation differs only in that pre-operative preparation cannot be so prolonged or extensive. The additional hazards of bleeding or perforation, such as infection and poor healing, are minimized by adequate replacement of blood and supportive antibiotics. It is our custom to give these patients penicillin, 500,000 units intramuscularly, and streptomycin, $0.5 \mathrm{~g}$. every 12 hours for seven days. Vitamins B, C and $\mathrm{K}$ are also given by injection on admission, as peptic ulcer cases are amongst the few types of acute vitamin deficiencies still seen in clinical practice.

\section{Some of the Complications and Their Management}

One should be on the look out for the complications that may follow partial gastrectomy, for their early recognition leads to easier control. Certain cases are associated with special risks: for example, the effects of severe haemorrhage on 
vital centres make cardiac, respiratory, and even cerebral complications a particular risk in emergency gastrectomy performed for bleeding. Tissue repair is slower as a consequence of the loss of protein and poor oxygenation, so that wound infection, haematoma and disruption are commoner. Many of these patients are elderly and in them post-operative bronchopneumonia, retention of urine with its danger of ascending infection, bed sores, faecal impaction and mental deterioration are additional risks to life.

Post-operative shock is unusual, but may follow if there has been much bleeding before or during operation. Blood or plasma transfusion, if started during operation, is continued as indicated.

\section{Haemorrhage}

Bleeding from the suture line is recognized early by the nature of the aspirations. The vomiting of bright red blood or large clots or continued bloody aspirations is associated with a rising pulse rate, pallor, sweating, restlessness, air hunger or melaena. Serious bleeding is rare and is usually the result of failure of ' all the coats' suture to control the bleeding vessels. Keeping the patient comfortable by correct hydration, sips of cold water, morphia and a slow blood transfusion in the majority of cases sees the bleeding ceasing spontaneously. However, if haemorrhage continues severely, the abdomen must be reopened and the gastric remnant exposed and inspected. An independent incision is made into an accessible part of the anterior stomach wall and the suture line inspected. A single bleeding point may be caught and tied or several bleeding points firmly undersewn with mattress sutures of catgut. The gastrotomy wound is carefully closed and the abdominal wound resutured. To illustrate how this complication can be minimized one of the staff, who has done most of the gastrectomies, has only once in over 3,000 cases had to reopen for bleeding after gastrectomy. On that occasion no bleeding point was found and the patient recovered. To the best of our knowledge, there has never been a death from suture-line bleeding since the clinic started.

Intraperitoneal bleeding from splenic capsular tears, slipped ligatures or from vessels in an ulcer crater which have not been ' under-run,' may be suspected if the pulse rate remains high. The abdominal signs of haemoperitoneum in the immediate post-operative period can be deceptively absent or masked by wound pain. Immediate blood transfusion and early exploration are carried out and the bleeding controlled. In our experience the bleeding has been from delayed splenic bleeding and on two occasions a late splenectomy had to be performed. (It is our practice to inspect the spleen at operation to ensure that no capsular $\frac{\overrightarrow{0}}{\mathrm{Q}}$ other injury has occurred.) Blood may collect also when many adhesions from previous operations ake divided.

\section{Obstruction of the Stoma}

This is recognized by excessive gastric aspirations and is more common after gastro-duodenal anastomosis than after gastro-jejunal. It may 1 se caused by oedema of the mucosa, particularly aft coarse stitching or in hypoproteinaemic patients who have had emergency operations. Other rare causes may be accidental suturing of the mucosa of the opposite side when completing the anterief ' all the coats' layer or stomal constriction from pressure of omental covering placed over the anastomosis. The tendency to stomal blockage b. oedema is countered during the operation by close stitching of the mucosal layers and by using at least half the diameter of the stomach tailored to fit the duodenum. A small longitudinal incision in the - anterior wall of the duodenum (illustrated) is iro dicated when the duodenal lumen is small or the gastric folds excessively thick; this allows the anterior suture line to lie at a different plane to the posterior line. The stoma is felt at the end of the operation to break away any mucosal attachment of the posterior wall to the anterior suture line Provided physiological balance is maintained 建 safe to await events and to keep the stomach en by hourly aspirations. If after Io days there isino progress, an X-ray examination is performed, minutes, one and three hours after injection of lipiodol into the stomach. If the obstruction absolute, operative correction is indicated. Fof this it may be necessary to mobilize the whole $\overrightarrow{0}$ the duodenum so that the anastomosis can b remade or widened; alternatively, a gastro jejun ostomy can be done. Obstruction to the lower oesophagus may rarely result following removal of a juxta-cardia gastric ulcer. During this procedure. a wide-bore stomach tube can be passed during operation to prevent encroaching too much on the oesophagus.

\section{Suture-Line Leakage and Post-operative Peritonitis}

Suture-line leakage, either from the duodenat. stump or from the anastomosis, may occur, but is fortunately, rare. Both can be prevented by careful and painstaking technique. Avoidance of long afferent loop liable to obstruct, twist of herniate, and ascertaining that the stoma lies without twist or kink, minimizes the risk of duof denal burst from the back pressure. Unsuspecte injury to the duodenum from sharp forceps or heavy handling may cause a duodenal leak -Adequate mobilization of the duodenum an $\$$ 


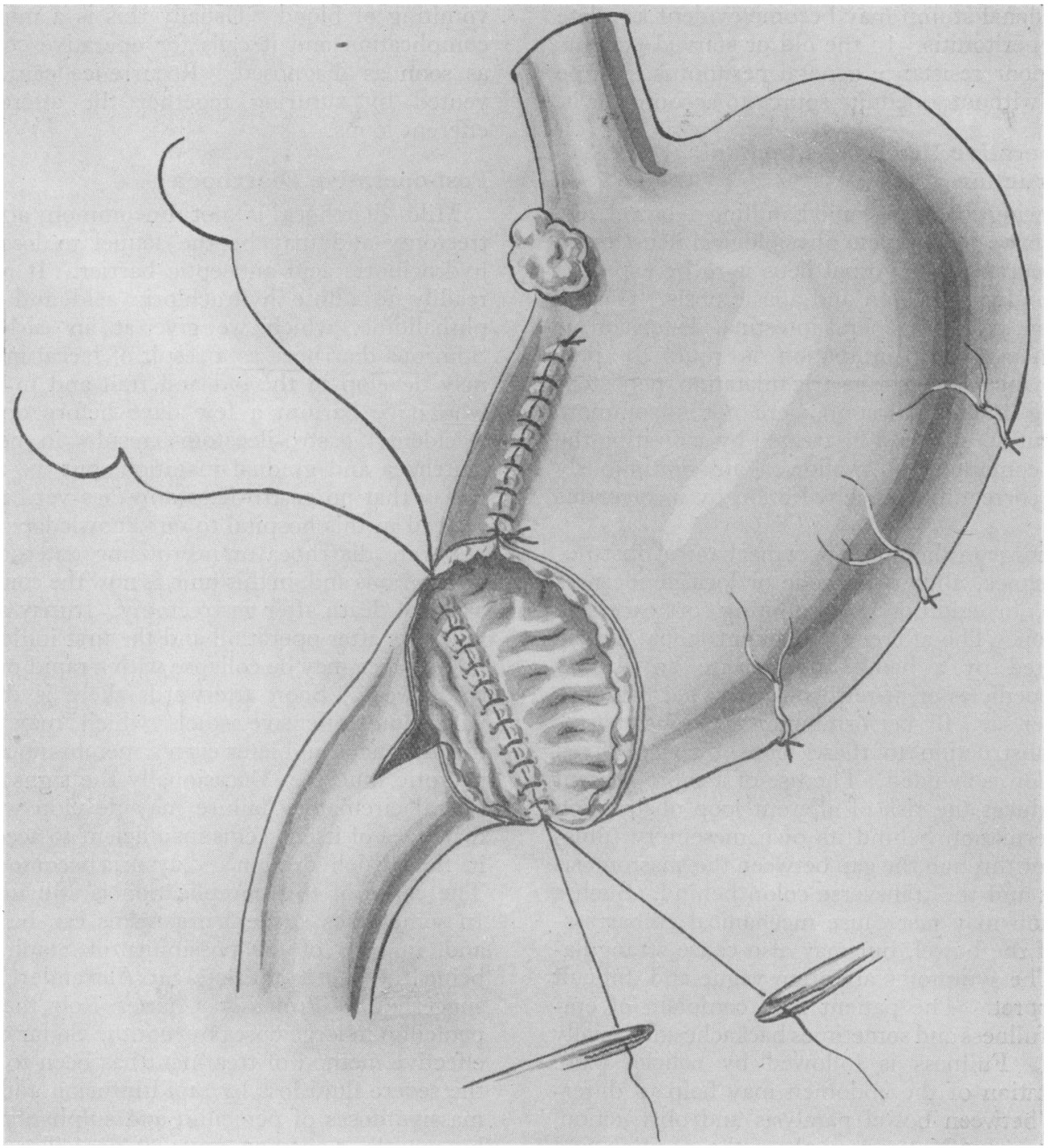

FIG. I.-To illustrate the method of enlarging the duodenum in the Billroth I Operation.

greater curve of the stomach allows a gastroduodenal anastomosis to be without tension. A leakage in the absence of a drain may lead to local or general peritonitis, and in such a case the abdomen should be explored and the fluid aspirated. If the leak is at the stoma, it must be repaired by suture and a rubber drain arranged to the site. If the duodenal stump is leaking, it should be drained and continuous gentle suction made from the drainage tube. The bile obtained, if bulky, should be returned to the stomach by the Ryle's tube. The skin of the abdominal wall is protected from the digestive enzymes by liberal applications of aluminium paste and the fluid balance maintained by intravenous drip of saline, blood and plasma. If a fistula persists, reoperation will be necessary with a view to anastomosis of afferent to efferent loops.

Peritonitis may also follow failure to drain an ulcer crater which lies over the pancreas and into which open small pancreatic duct radicals. Pooling of blood contaminated during operation may initiate the development of subphrenic abscesses which may develop into general peritonitis. In a very fat patient it is advisable to remove the great omentum lest extensive fat necrosis should follow its devascularization. Accidental injury to the midcolic vessels or to the hepatic flexure when closing 
the duodenal stump may become evident as a late general peritonitis. In the old or starved patients with a poor resistance general peritonitis may be present without a definite source to account for it.

\section{Post-operative Ileus and Mechanical Obstruction}

Following coeliotomy and handling of bowel, one usually expects complete physiological ileus for 36 to 48 hours. This normal ileus is to be expected more so after resection and anastomosis. Gastric dilatation, or even general intestinal distension, is rare when gastric intubation is routinely performed; before its use gastric dilatation, persistent vomiting and dehydration were not uncommon. Persistent ileus should be treated by aspirating the gastric contents and swallowed air continuously and by correcting the dehydration by intravenous fluids.

The surgeon should suspect mechanical obstruction if shock, abdominal colic or local tenderness develop in addition to vomiting or excessive aspiration. The afferent or efferent loops may be obstructed by a band, adhesion to an abscess around pedicles or, if retrocolic, may herniate into the lesser sac. By performing an antecolic anastomosis obstruction to these loops by an unfixed mesocolon is avoided. The use of a short afferent loop reduces the risk of afferent loop obstruction from herniation behind its own mesentery (illustrated) or through the gap between the anastomosis in front and the transverse colon behind. Such a herniation may just cause mechanical embarrassment of the bowel, but may also cause strangulation. The symptoms are often vague and difficult to interpret. The patient may complain of epigastric fullness and sometimes backache and usually vomits. Fullness is followed by colicky pain. Auscultation of the abdomen may help to differentiate between bowel paralysis and obstruction, whilst a scout $\mathrm{X}$-ray may show the position and extent of any dilated, obstructed bowel. Absence of bile from the gastric aspirate makes one suspect obstruction of the afferent loop. This is sometimes relieved by turning the patient on to one or other side with the foot of the bed raised and relief may be confirmed by the diminution of pain and reappearance of bile in the gastric contents. Acute pancreatitis should be excluded by a serum amylase estimation, though the amylase may also be raised in afferent loop obstruction. If no relief is obtained, laparotomy should be performed without delay. Internal herniation is reduced and the gap closed and measures taken to prevent a recurrence. If reduction is impossible, the loops should be short-circuited. Retrograde jejuno-gastric intussusception as a cause of post-operative obstruction is suspected if epigastric colic is associated with the vomiting of blood. Usually this is a much later complication- and it calls for operative correction as soon as diagnosed. Recurrence can be pre- $\frac{\pi}{\infty}$ vented by suturing together the afferent and $\stackrel{\complement}{c}$ efferent loops.

\section{Post-operative Diarrhoea}

Mild diarrhoea is not uncommon after gas- 듬 trectomy and may be the sequel to loss of the $\frac{\bar{c}}{\bar{c}}$ hydrochloric acid antiseptic barrier. It responds $\underset{\Omega}{\widehat{\Phi}}$ readily to dilute hydrochloric acid and sulphaphthalidine, which we give at an early stage. ${ }^{\infty}$ Spurious diarrhoea as a result of faecal impaction $\vec{\circ}$ may develop in the old and frail and in patients $\overrightarrow{\vec{\omega}}$ who have barium a few days before operation. $\mathscr{\omega}$ Accidental gastro-ileostomy results in persistent diarrhoea and gradual inanition, but we are glad 3 . to say that no gastro-ileostomy has yet been performed in this hospital to our knowledge.

Severe diarrhoea or necrotizing enterocolitis is very serious and, in this unit, is now the commonest $+\infty$ cause of death after gastrectomy. It may appear $\mathrm{a}_{\circ}$ few days after operation and the first indication of $\stackrel{\circ}{\supset}$ its presence may be collapse with a rapid pulse and $\vec{T}_{7}$ cold sweat. Soon afterwards there is diarrhoea ${ }_{0}^{\circ}$ with fluid offensive stools which may contain blood, mucus and later even a membranous cast of $\stackrel{\hat{2}}{2}$ necrotic mucosa. Occasionally the signs of peri $-\overrightarrow{0}$ pheral circulatory failure may develop when tte of diarrhoea of itself seems insufficient to account f( it. Severe dehydration and dysuria become market. The cause of this complication is still unknown. In some cases virulent organisms can be isolated and, in view of the possibility of Staph. aureus $\frac{\partial}{\varnothing}$ being the cause, the late Sir Alexander Fleming $\propto$ suggested to Professor Charles Rob the use of $\overrightarrow{\hat{O}}$ penicillin in large doses by mouth. So far our most 3 effective method of treatment has been to counter the severe fluid loss by rapid infusion and to give? massive doses of penicillin and sulphaphthalidine음 by mouth, 5,000,000 units of penicillin (crystal-3. line) dissolved in water, followed by $2,000,000 \frac{3}{3}$ units two-hourly, as well as cremorthalidine in adequate doses. One patient was found to have sloughed a length of mucous membrane of the terminal ileum into an abscess cavity around the $\mathrm{O}$ bowel and made an eventual full recovery following $>$ resection of the necrotic bowel. As this condition may be related to the loss of the hydrochloric acidNo antiseptic barrier, we are, at St. James's, at present trying out the effect of giving dilute hydrochloric ${ }_{N}$ acid to all post-gastrectomy cases for one week $\omega$ after operation.

\section{Late Complications}

These include gastro-jejunal or stomal ulcer, gastrocolic fistula and post-cibal symptoms.

These complications are, fortunately, rare and $\frac{\mathscr{D}}{\stackrel{\circ}{D}}$ 
usually present with symptoms within two years of operation. Patients who develop stomal ulcers will complain of post-prandial pain relieved by alkalis, or they may bleed or perforate. Penetration of the colon may cause a gastro-jejuno-colic fistula to be established. This causes marked deterioration as the gastric content passes directly into the colon and the diarrhoea is made worse by faecal material irritating the small bowel. As a result of loss of fluid and food, dehydration, starvation and vitamin deficiencies may be severe. Treatment of uncomplicated stomal ulcer is by vagotomy with refashioning of the stoma if the distortion from fibrosis is great. Gastro-jejuno-colic fistula is best treated in stages, the first operation being diversion of the faecal stream by proximal colostomy.

\section{Post-cibal Disturbances}

About 60 to 70 per cent. of patients will complain of unpleasant symptoms after eating for the first few weeks or months after partial gastrectomy. In the majority these symptoms are mild and in- clude a feeling of excessive warmth, sweating, dizziness, epigastric discomfort and fatigue. These patients are advised to eat slowly but well, particularly of protein and fatty foods, to achieve a stable blood sugar level. Distension of the gastric remnant is less when the food is taken dry and fluids taken between meals. A rest, reclining for about 20 minutes after food, is also advised. In severe cases with persistent symptoms for over a year a further operation, such as a conversion to a gastro-duodenal anastomosis, or rarely to a Rouxen-Y gastrectomy, if bilious vomiting is excessive, may be effective in controlling these symptoms.

\section{Acknowledgment}

I wish to record my indebtedness to Sister Jackson and Sister Ridgway, who have nursed so many of our patients and upon whose invaluable experience $I$ have drawn in preparing this article. I am very grateful to $\mathrm{Mr}$. Norman Tanner for his advice and constant help.

\section{HEPATIC DISEASE}

\section{(Postgraduate Medical Journal, October, 1956)}

Price: 3s. 9d. post free

\section{JAUNDICE}

Sheila Sherlock, M.D., M.R.C.P.

HEPATIC COMA

J. M. Walshe, M.A., M.R.C.P.

\section{SURGICAL TREATMENT OF PORTAL HYPERTENSION}

A. I. S. Macpherson, Ch M., F.R.C.S.E.

WILSON'S DISEASE

A. G. Bearn, M.D.
ASCITES IN LIVER DISEASE

Michael Atkinson, M.D. (Lond.)

M.R.C.P.

\section{PSYCHIATRIC ASPECTS OF LIVER} DISEASE

Esther A. Davidson, M.R.C.P.Ed., and

W. H. J. Summerskill, M.A., M.R.C.P.

\section{PERCUTANEOUS PORTAL} VENOGRAPHY

David Sutton, M.D., M.R.C.P., F.F.R.

Published by

THE FELLOWSHIP OF POSTGRADUATE - MEDICINE

60, Portland Place, London, W.1 